\title{
TRANSCENDING CLASSICAL INVARIANT THEORY
}

\author{
ROGER HOWE
}

\section{INTRODUCTION}

Let $\mathrm{Sp}_{2 n}(\mathbf{R})=\mathrm{Sp}_{2 n}=\mathrm{Sp}$ be the real symplectic group of rank $n$. Let $\widetilde{\mathrm{Sp}}$ denote the two-fold cover of $\mathrm{Sp}$. There is a unitary representation, constructed by Shale [Sh] and Weil [WA], of $\widetilde{\mathrm{Sp}}$ that is of considerable interest in the theory of automorphic forms. We denote this representation by $\omega$ and call it the oscillator representation. The purpose of this paper is to establish about $\omega$ a fact that should be useful for clarifying the structure of spaces of automorphic forms constructed using $\omega$.

We begin by formulating the result. Let $E$ be a reductive subgroup of $\mathrm{Sp}$, and let $\widetilde{E}$ denote the inverse image of $E$ in $\mathrm{Sp}$. Denote by $\mathscr{R}(\widetilde{E})$ the set of infinitesimal equivalence classes of continuous irreducible admissible representations of $\widetilde{E}$ on locally convex spaces. Let $\omega^{\infty}$ be the smooth representation of $\widetilde{\mathrm{Sp}}$ associated to $\omega$. Let $\omega$ be realized on a Hilbert space $\mathscr{Y}$, and let the subspace of smooth vectors, on which space $\omega^{\infty}$ is defined, be written $\mathscr{Y}^{\infty}$. Denote by $\mathscr{R}(\widetilde{E}, \omega)$ the set of elements of $\mathscr{R}(\widetilde{E})$ which are realized as quotients by $\omega^{\infty}(\widetilde{E})$-invariant closed subspaces of $\mathscr{Y}^{\infty}$.

Consider a reductive dual pair $\left(G, G^{\prime}\right) \subseteq \mathrm{Sp}$ [H2]. It is not hard to show that $\widetilde{G}$ and $\widetilde{G}^{\prime}$ commute with one another. Hence, we may regard $\omega^{\infty} \mid \widetilde{G} \cdot \widetilde{G}^{\prime}$ as a representation of $\widetilde{G} \times \widetilde{G}^{\prime}$. It is well known $[\mathrm{F}]$ that $\mathscr{R}\left(\widetilde{G} \times \widetilde{G}^{\prime}\right) \simeq \mathscr{R}(\widetilde{G}) \times \mathscr{R}\left(\widetilde{G}^{\prime}\right)$. The identification associates to $\rho \in \mathscr{R}(\widetilde{G})$ and $\rho^{\prime} \in \mathscr{R}\left(\widetilde{G}^{\prime}\right)$ the tensor product $\rho \otimes \rho^{\prime}$. (We note that $\rho \otimes \rho^{\prime}$ is not defined as a topological vector space; nevertheless, the infinitesimal equivalence class of $\rho \otimes \rho^{\prime}$ is well defined.) Select $\rho \otimes \rho^{\prime} \in \mathscr{R}\left(\widetilde{G} \times \widetilde{G}^{\prime}\right)$. Suppose that, in fact, $\rho \otimes \rho^{\prime} \in \mathscr{R}\left(\widetilde{G} \cdot \widetilde{G}^{\prime}, \omega\right)$. Then clearly $\rho \in \mathscr{R}(\widetilde{G}, \omega)$ and $\rho^{\prime} \in \mathscr{R}\left(\widetilde{G}^{\prime}, \omega\right)$. Hence, $\mathscr{R}\left(\widetilde{G} \cdot \widetilde{G}^{\prime}, \omega\right)$ defines the graph of a correspondence between certain subsets of $\mathscr{R}(\widetilde{G}, \omega)$ and $\mathscr{R}\left(\widetilde{G}^{\prime}, \omega\right)$. In fact, the situation is quite precise.

Theorem 1. The set $\mathscr{R}\left(\widetilde{G} \cdot \widetilde{G}^{\prime}, \omega\right)$ is the graph of a bijection between (all of) $\mathscr{R}(\widetilde{G}, \omega)$ and (all of $) \mathscr{R}\left(\widetilde{G}^{\prime}, \omega\right)$. Moreover, an element $\rho \otimes \rho^{\prime}$ of $\mathscr{R}\left(\widetilde{G} \cdot \widetilde{G}^{\prime}, \omega\right)$ occurs as a quotient of $\omega^{\infty}$ in a unique way.

Received by the editors November 18, 1988.

1980 Mathematics Subject Classification (1985 Revision). Primary 22E45. 
The proof of Theorem 1 will be given in $\S \S 2-5$. Here we give a variant formulation of the result and some comments.

Given $\rho \in \mathscr{R}(\widetilde{G}, \omega)$, it probably will be realizable as a quotient of $\mathscr{Y}^{\infty}$ in more than one way. That is, there may be more than one closed $\widetilde{G}$-invariant subspace $\mathscr{Y}_{1} \subseteq \mathscr{Y}^{\infty}$ such that $\mathscr{Y}^{\infty} / \mathscr{Y}_{1} \simeq \rho$. Let $\mathscr{Y}_{\rho}$ denote the intersection of all such spaces $\mathscr{Y}_{1}$. Then $\mathscr{Y}^{\infty} / \mathscr{Y}_{\rho}$ will not be simply a $\widetilde{G}$ module, but a $\widetilde{G} \cdot \widetilde{G}^{\prime}$ module. It is easy to see that we can write

$$
\mathscr{Y}^{\infty} / \mathscr{Y}_{\rho} \simeq \rho \otimes \rho_{1}^{\prime}
$$

where $\rho_{1}^{\prime}$ is a $\widetilde{G}^{\prime}$ module. Theorem 1 states that $\rho_{1}^{\prime}$ has a unique irreducible quotient. It is of interest to know as much as possible about the structure of $\rho_{1}^{\prime}$. We can, in fact, say a little more about it.

Let $E$ be a connected reductive Lie group and $\rho$ a representation of $E$. Recall that Harish-Chandra calls $\rho$ quasisimple if $\mathscr{Z} \mathscr{U}(\mathscr{E})$, the center of the universal enveloping algebra of the Lie algebra $\mathscr{E}$ of $E$, acts by scalar operators on the space of $\rho$. Some members of dual pairs are not connected. If $E$ is a nonconnected reductive Lie group, let $\mathscr{Z} \mathscr{U}(\mathscr{E})^{0}$ denote the subalgebra of $\mathscr{Z} \mathscr{U}(\mathscr{E})$ whose elements are invariant under the adjoint action $\operatorname{Ad} E$ (and not merely under the adjoint action of the identity component of $E$ ). Then a representation $\rho$ of $E$ will be called quasisimple if $\rho$ sends $\mathscr{Z} \mathscr{U}(\mathscr{E})^{0}$ to scalar operators.

Theorem 1A. The representation $\rho_{1}^{\prime}$ is a finitely generated admissible quasisimple representation of $\widetilde{G}^{\prime}$. Furthermore, $\rho_{1}^{\prime}$ has a unique irreducible $\widetilde{G}^{\prime}$ quotient $\rho^{\prime}$ so that $\rho \otimes \rho^{\prime} \in \mathscr{R}\left(\widetilde{G} \cdot \widetilde{G}^{\prime}, \omega\right)$.

Remarks. (a) In this result, "finitely generated" means that if $\widetilde{K}^{\prime}$ is a maximal compact subgroup of $\widetilde{G}^{\prime}$, then the $\widetilde{K}^{\prime}$-finite vectors in $\rho_{1}^{\prime}$ are finitely generated as a $\mathscr{U}\left(g^{\prime}\right)$-module.

(b) If $G$ or $G^{\prime}$ is compact, then Theorems 1 and $1 \mathrm{~A}$ are already known and have been treated by several authors [G, GK, H1, KV, Sa]. In fact, they then essentially amount to a version of Classical Invariant Theory [H1, H2, WH]. This special case will be a stepping stone to the general result.

(c) An analogue of Theorem $1 \mathrm{~A}$ in which one takes $\rho$ unitary and looks not at quotients of $\rho_{1}^{\prime}$ but at unitary subquotients has been considered and established in some cases by Rallis [R]. It is possible for $\rho_{1}^{\prime}$ to have unitary constituents when the quotient $\rho^{\prime}$ is not unitary. It is of interest to describe these.

(d) As we will see in $\S 6$ (Theorem 6.1), Theorem 1 has an $L^{2}$-version which may be formulated in terms of von Neumann algebras. 
I would like to thank Tom Enright for posing a catalytic problem and M. Takesaki for providing a reference in $\S 6$.

\section{Algebraic formulation}

Theorems 1 and $1 \mathrm{~A}$ are in some sense distribution theoretic. However, the proofs we give for them below are purely algebraic. We therefore begin by stating yet another, this time suitably algebraic, version of these results.

Let $K$ and $K^{\prime}$ be maximal compact subgroups of $G$ and $G^{\prime}$, and let $\mathfrak{g}$ and $\mathfrak{g}^{\prime}$ be the Lie algebras of $G$ and $G^{\prime}$. We will work in the standard current formalism of $(\mathfrak{g}, \widetilde{K})$ modules. Recall [D] that a $(\mathfrak{g}, \widetilde{K})$ module is a vector space $V$ which

(a) is simultaneously a module for $\mathfrak{g}$ and for $\widetilde{K}$;

(b) is a direct sum of subspaces invariant and irreducible under $\widetilde{K}$;

(c) is such that the actions of $\mathfrak{g}$ and $\widetilde{K}$ are compatible, i.e.,

(i) the differential of the $\widetilde{K}$ action is given by the inclusion of $\mathfrak{k}$, the Lie algebra of $\widetilde{K}$ in $\mathfrak{g}$, and

(ii) the action of $\widetilde{K}$ on End $V$ by conjugation normalizes the image of $\mathfrak{g}$ in End $V$ and induces the adjoint action of $\widetilde{K}$ on $\mathfrak{g}$.

The point is, of course, that $(\mathfrak{g}, \widetilde{K})$ modules are algebraic versions of $\widetilde{G}$ modules. It is an old theorem of Harish-Chandra [D] that if $\rho$ is an irreducible quasisimple representation of $\widetilde{G}$, then the $\widetilde{K}$-finite vectors in the space of $\rho$ define an irreducible $(\mathfrak{g}, \widetilde{K})$ module, and all irreducible $(\mathfrak{g}, \widetilde{K})$ modules arise in this way.

The maximal compact subgroup of $\mathrm{Sp}=\mathrm{Sp}_{2 n}$ is $\mathrm{U}=\mathrm{U}_{n}$, the unitary group in $n$ variables. Let $\mathfrak{s p}$ be the Lie algebra of $\mathrm{Sp}$, and $\mathfrak{u}$ the Lie algebra of $U$. Let

$$
\mathfrak{s p}=\mathfrak{u} \oplus \mathfrak{q}
$$

be the Cartan decomposition of $\mathfrak{s p}$. The $(\mathfrak{s p}, \tilde{\mathrm{U}})$ module associated to $\omega$ is made explicit by the realization of $\omega$ known as the Fock model [C, H3, Sg]. Using it, one sees that the $\widetilde{U}$-finite vectors in $\omega$ form a space isomorphic to $\mathscr{P}\left(\mathbf{C}^{n}\right)=\mathscr{P}_{n}=\mathscr{P}$, the space of polynomials on $\mathbf{C}^{n}$. When this identification is made, we have

$$
\begin{aligned}
\omega\left(\mathfrak{s p}_{\mathbf{C}}\right) & =\mathfrak{s p}^{(1,1)} \oplus \mathfrak{s p}^{(2,0)} \oplus \mathfrak{s p}^{(0,2)}, \\
\mathfrak{s p}^{(1,1)} & =\text { span of }\left\{\frac{1}{2}\left(z_{i} \frac{\partial}{\partial z_{j}}+\frac{\partial}{\partial z_{j}} z_{i}\right)\right\}, \\
\mathfrak{s p}^{(2,0)} & =\text { span of }\left\{z_{i} z_{j}\right\}, \\
\mathfrak{s p}^{(0,2)} & =\text { span of }\left\{\frac{\partial^{2}}{\partial z_{i} \partial z_{j}}\right\} .
\end{aligned}
$$

Here $\mathfrak{s p}_{\mathbf{C}}$ indicates the complexification of $\mathfrak{s p}$. The indices $i$ and $j$ vary from 1 to $n$. Note that while $\mathfrak{s p}$ is a real Lie algebra, the $\mathfrak{s p}^{(a, b)}$ are complex 
subalgebras of $\mathfrak{s p}_{\mathfrak{C}}$. The relation between decompositions (2.1) and (2.2) is

$$
\begin{aligned}
& \omega\left(\mathfrak{u}_{\mathbf{C}}\right)=\mathfrak{s p}^{(1,1)}, \\
& \omega\left(\mathfrak{q}_{\mathbf{C}}\right)=\mathfrak{s p}^{(2,0)} \oplus \mathfrak{s p}^{(0,2)} .
\end{aligned}
$$

We may suppose $G$ and $G^{\prime}$ are embedded in $\mathrm{Sp}$ in such a way that the Cartan decomposition (2.1) of $\mathfrak{s p}$ also induces Cartan decompositions of $\mathfrak{g}$ and $\mathfrak{g}^{\prime}$. Thus,

$$
\begin{aligned}
\mathfrak{g}=\mathfrak{k} \oplus \mathfrak{p}, & \mathfrak{k}=\mathfrak{u} \cap \mathfrak{g}, & \mathfrak{p}=\mathfrak{q} \cap \mathfrak{g}, \\
\mathfrak{g}^{\prime}=\mathfrak{k}^{\prime} \oplus \mathfrak{p}^{\prime}, & \mathfrak{k}^{\prime}=\mathfrak{u} \cap \mathfrak{g}^{\prime}, & \mathfrak{p}^{\prime}=\mathfrak{q} \cap \mathfrak{g}^{\prime} .
\end{aligned}
$$

Then $\mathscr{P}$ is a $(\mathfrak{g}, \widetilde{K})$ module and a $\left(\mathfrak{g}^{\prime}, \widetilde{K}^{\prime}\right)$ module. We can define the analogue of $\mathscr{R}(\widetilde{G}, \omega)$. Let $\mathscr{R}(\mathfrak{g}, \widetilde{K}, \omega)$ denote the set of isomorphism classes of irreducible $(\mathfrak{g}, \widetilde{K})$ modules which can be realized in the form $\mathscr{P} / \mathcal{N}$, where $\mathscr{N} \subseteq \mathscr{P}$ is a $\mathfrak{g}$ and $\widetilde{K}$-invariant subspace. For $\rho \in \mathscr{R}(\mathfrak{g}, \widetilde{K}, \omega)$, let $\mathscr{N}_{\rho}$ be the intersection of all subspaces $\mathscr{N} \subseteq \mathscr{P}$ such that $\mathscr{P} / \mathscr{N}$ is a $(\mathfrak{g}, \widetilde{K})$ module isomorphic to $\rho$. Then $\mathscr{P} / \mathscr{N}_{\rho}$ is a $\left(\mathfrak{g} \oplus \mathfrak{g}^{\prime}, \widetilde{K} \cdot \widetilde{K}^{\prime}\right)$ module and has the form

$$
\mathscr{P} / \mathscr{N}_{\rho} \simeq \rho \otimes \rho_{1}^{\prime},
$$

where $\rho_{1}^{\prime}$ is a $\left(\mathfrak{g}^{\prime}, \widetilde{K}^{\prime}\right)$ module.

Theorem 2.1. The $\left(\mathfrak{g}^{\prime}, \widetilde{K}^{\prime}\right)$ module $\rho_{1}^{\prime}$ is finitely generated, admissible, and quasisimple. Furthermore, $\rho_{1}^{\prime}$ has a unique irreducible $\left(\mathfrak{g}^{\prime}, \widetilde{K}^{\prime}\right)$ quotient $\rho^{\prime}$, and the correspondence $\rho \rightarrow \rho^{\prime}$ defines a bijection from $\mathscr{R}(\mathfrak{g}, \widetilde{K}, \omega)$ to $\mathscr{R}\left(\mathfrak{g}^{\prime}, \widetilde{K}^{\prime}, \omega\right)$.

It is actually this theorem which will be proved in this and the next three sections. Our analysis will reveal some further information about the bijection $\rho \leftrightarrow \rho^{\prime}$. These supplementary facts will be pointed out as they arise. Before beginning the proof of Theorem 2.1, we will show it yields Theorem 1 .

Lemma 2.2. Theorems 1 and 1A follow from Theorem 2.1.

Proof. The main point is simply that $\mathscr{P}$ is dense in $\mathscr{Y}$. Choose $\rho \in \mathscr{R}(\widetilde{G}, \omega)$. Let $\rho^{0}$ be the associated $(\mathfrak{g}, \widetilde{K})$ module defined by the $\widetilde{K}$-finite vectors. Let $\mathscr{Y}_{1} \subseteq \mathscr{Y}^{\infty}$ be a $\widetilde{G}$-invariant subspace such that $\mathscr{Y}^{\infty} / \mathscr{Y}_{1} \simeq \rho$. Then the image of $\mathscr{P}$ in $\mathscr{Y}^{\infty} / \mathscr{Y}_{1}$ is dense, and the projection $\mathscr{P} \rightarrow \mathscr{Y}^{\infty} / \mathscr{Y}_{1}$ is a homomorphism of $(\mathfrak{g}, \widetilde{K})$ modules. Since the $K$-isotypic components of $\rho$ are finite dimensional, it follows that $\mathscr{P} / \mathscr{P} \cap \mathscr{Y}_{1} \simeq \rho^{0}$. Hence, $\rho^{0} \in \mathscr{R}(\mathfrak{g}, \widetilde{K}, \omega)$. Also $\mathscr{P} \cap \mathscr{Y}_{1} \supseteq \mathscr{N}_{\rho^{0}}$. Therefore, the quotient map $\mathscr{Y}^{\infty} \rightarrow \mathscr{Y}^{\infty} / \mathscr{Y}_{\rho}$ defines a map

$$
\mathscr{P} / \mathscr{N}_{\rho^{0}} \rightarrow \mathscr{Y}^{\infty} / \mathscr{Y}_{\rho}
$$

with dense image. Since by Theorem 2.1 the left-hand side of (2.6) is admissible as a $\left(\mathfrak{g} \oplus \mathfrak{g}^{\prime}, \widetilde{K} \cdot \widetilde{K}^{\prime}\right)$ module, the $\widetilde{K} \cdot \widetilde{K}^{\prime}$-isotypic subspaces are finite dimensional. Since the mapping (2.6) has dense image, the $\widetilde{K} \cdot \widetilde{K}$-isotypic subspaces on the right-hand side must also be finite dimensional, and the mapping (2.6) must 
carry $\mathscr{P}$ onto the space of $\widetilde{K} \cdot \widetilde{K}^{\prime}$-finite vectors in $\mathscr{Y}^{\infty} / \mathscr{Y}_{\rho}$. Hence, $\mathscr{Y}^{\infty} / \mathscr{Y}_{\rho}$ is finitely generated, admissible, and quasisimple. Furthermore, if $\chi$ is any irreducible $\widetilde{G}^{\prime}$-quotient of $\rho_{1}^{\prime}$, then the $\widetilde{K}^{\prime}$-finite vectors in $\chi$ must be a quotient of $\left(\rho_{1}^{\prime}\right)^{0}$, and, therefore, $\chi$ is uniquely determined by Theorem 2.1. This proves Lemma 2.2.

Since $\widetilde{G}$ modules will not enter the discussion from here on, we will no longer

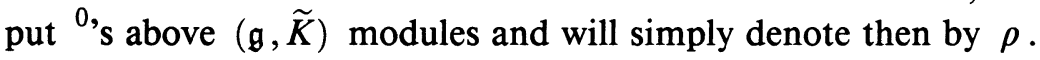

The proof of Theorem 2.1 is not difficult. It follows by general considerations from a few key facts about the structure of $\mathscr{P}$ as a $\left(\mathfrak{g} \oplus \mathfrak{g}^{\prime}, \widetilde{K} \cdot \widetilde{K}^{\prime}\right)$ module. These structural results follow in turn from some remarkable relationships between $\left(\mathfrak{g}, \mathfrak{g}^{\prime}\right)$ and three other dual pairs in $\mathfrak{s p}$. In an attempt to make the structure of the argument as evident as possible, we have divided it into three parts. In $\S 3$, we will state the facts we need about dual pairs, giving the basic pair $\left(\mathfrak{o}_{p, q}, \mathfrak{s p}_{2 n}\right)$ as an example, and we will use these facts to establish the relevant structural results about $\mathscr{P}$. These will be used in $\S 4$ to deduce Theorem 2.1. Then $\S 5$ will be devoted to proving the facts about dual pairs stated in $\S 3$.

\section{Structure of $\mathscr{P}$ as $\mathfrak{g} \oplus \mathfrak{g}^{\prime}$ MOdule}

Let $\left(\mathfrak{g}, \mathfrak{g}^{\prime}\right) \subseteq \mathfrak{s p}$ be a reductive dual pair. Let $\mathfrak{k}$ and $\mathfrak{k}^{\prime}$ be the Lie algebras of the maximal compact subgroups $K$ and $K^{\prime}$ of $G$ and $G^{\prime}$. Let $\mathfrak{k}$ and $\mathfrak{k}^{\prime}$ be chosen as in $\S 2$ so that the Cartan decompositions of $\mathfrak{g}$ and $\mathfrak{g}^{\prime}$ are compatible with that of $\mathfrak{s p}$, as in equation (2.4).

Example. Let $\mathbf{R}^{p+q}$ be endowed with the indefinite inner product

$$
\sum_{j=1}^{p} x_{j}^{2}-\sum_{j=p+1}^{p+q} x_{j}^{2}
$$

Let $\mathbf{R}^{2 n}$ be endowed with the symplectic form

$$
\left\langle(x, y),\left(x^{\prime}, y^{\prime}\right)\right\rangle=\sum_{i=1}^{n} x_{i} y_{i}^{\prime}-x_{i}^{\prime} y_{i} .
$$

Then on $\mathbf{R}^{2 n(p+q)} \simeq \mathbf{R}^{p+q} \otimes \mathbf{R}^{2 n}$ we can define a symplectic form to be the tensor product of the forms on $\mathbf{R}^{p+q}$ and $\mathbf{R}^{2 n}$. Clearly, the isometry groups $\mathrm{O}_{p, q}$ and $\mathrm{Sp}_{2 n}$ will act on $\mathbf{R}^{2 n(p+q)}$ by acting on the appropriate factor $\mathbf{R}^{p+q}$ or $\mathbf{R}^{2 n}$, and will preserve the symplectic form. They clearly also commute with each other. The pair $\left(\mathrm{O}_{p, q}, \mathrm{Sp}_{2 n}\right)$ forms a reductive dual pair in $\mathrm{Sp}_{2 n(p+q)}$, and the pair $\left(\mathfrak{o}_{p, q}, \mathfrak{s p}_{2 n}\right)$ of their Lie algebras forms a dual pair in $\mathfrak{s p}_{2 n(p+q)}$. The maximal compact subgroup of $\mathrm{O}_{p, q}$ is $\mathrm{O}_{p} \times \mathrm{O}_{q}$, and the maximal compact subgroup of $\mathrm{Sp}_{2 n}$ is, as we have already noted, the unitary group $\mathrm{U}_{n}$.

Fact 1 . The Lie algebras $\mathfrak{k}$ and $\mathfrak{k}^{\prime}$ are themselves members of reductive dual pairs $\left(\mathfrak{k}, \mathfrak{m}^{\prime}\right)$ and $\left(\mathfrak{m}, \mathfrak{k}^{\prime}\right)$. 
Thus, we have sequences of inclusions

$$
\mathfrak{k} \subseteq \mathfrak{g} \subseteq \mathfrak{m}, \quad \mathfrak{m}^{\prime} \supseteq \mathfrak{g}^{\prime} \supseteq \mathfrak{k}^{\prime}
$$

and the pairs of similarly placed algebras form dual pairs.

Example. For the pair $\left(\mathfrak{o}_{p, q}, \mathfrak{s p}_{2 n}\right)$ we have, if $p q \neq 0, \mathfrak{m}^{\prime}=\mathfrak{s p}_{2 n} \times \mathfrak{s p}_{2 n}$ and $\mathfrak{m}=\mathfrak{u}_{p, q}$, the indefinite unitary group of signature $p, q$.

Since $\mathfrak{k} \subseteq \mathfrak{u}$, adk preserves each of the spaces $\mathfrak{s p}^{(a, b)}$ of equation (2.2). Since $\mathfrak{m}^{\prime}$ is the full centralizer of $\mathfrak{k}$ in $\mathfrak{s p}$, we have the decompositions

$$
\begin{array}{cc}
\mathfrak{m}_{\mathbf{C}}^{\prime}=\mathfrak{m}^{(1,1)} \oplus \mathfrak{m}^{(2,0)} \oplus \mathfrak{m}^{(0,2)}, & \mathfrak{m}^{(i, j)}=\mathfrak{m}_{\mathbf{C}}^{\prime} \cap \mathfrak{s p}^{(i, j)}, \\
\mathfrak{m}_{\mathbf{C}}=\mathfrak{m}^{(1,1)} \oplus \mathfrak{m}^{(2,0)} \oplus \mathfrak{m}^{(0,2)}, & \mathfrak{m}^{(i, j)}=\mathfrak{m}_{\mathbf{C}} \cap \mathfrak{s p}{ }^{(i, j)} .
\end{array}
$$

The decomposition (3.2) is a refinement of the (complexified) Cartan decomposition. In particular, $\mathfrak{m}^{\prime(1,1)}$ is the complexification of the Lie algebra of the maximal compact subgroup of $M^{\prime}$, the group with Lie algebra $\mathfrak{m}^{\prime}$. On the other hand, $\mathfrak{m}^{\prime(1,1)}$ is the centralizer of $\mathfrak{k}$ in $\mathfrak{s p}^{(1,1)}$. Hence, $\mathfrak{m}^{(1,1)}$ must be a product of $\mathfrak{g l}$ Lie algebras, or $\mathfrak{m}^{(1,1)} \cap \mathfrak{s p}=\mathfrak{m}_{0}^{(1,1)}$, a real form of $\mathfrak{m}^{\prime(1,1)}$, is a product of unitary Lie algebras.

Fact 2. The pair $\left(\mathfrak{m}_{0}^{(1,1)}, \mathfrak{m}_{0}^{(1,1)}\right)$ is a dual pair in $\mathfrak{s p . ~}$

Example. With the pair $\left(\mathfrak{o}_{p, q}, \mathfrak{s p}_{2 n}\right)$, one has $\mathfrak{m}=\mathfrak{u}_{p, q}$ and $\mathfrak{m}^{\prime}=\mathfrak{s p}_{2 n} \times \mathfrak{s p}_{2 n}$. Thus, $\mathfrak{m}_{0}^{\prime(1,1)} \simeq \mathfrak{u}_{n} \times \mathfrak{u}_{n}$, and $\mathfrak{m}_{0}^{(1,1)} \simeq \mathfrak{u}_{p} \times \mathfrak{u}_{q}$. Thus, $\left(\mathfrak{m}_{0}^{(1,1)}, \mathfrak{m}^{(1,1)}\right)$ is a direct sum of the dual pairs $\left(\mathfrak{u}_{p}, \mathfrak{u}_{n}\right) \in \mathfrak{s p}_{2 n p}$, and $\left(\mathfrak{u}_{q}, \mathfrak{u}_{n}\right) \subseteq \mathfrak{s p}_{2 n q}$.

Thus, we can expand the sequence (3.1) to

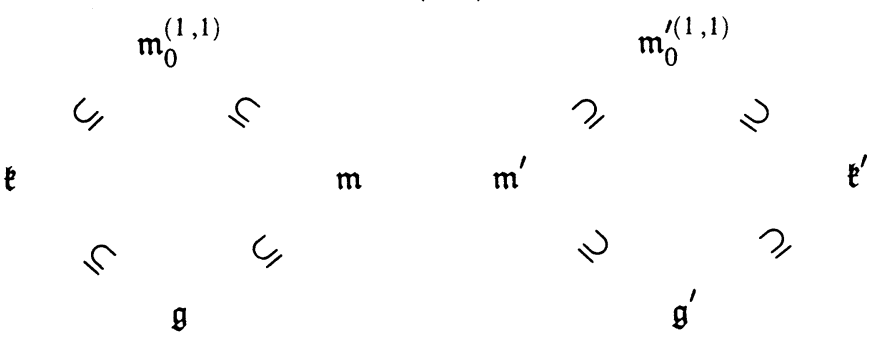

The pairs of Lie algebras similarly placed in the two diamonds are dual pairs.

Note that $\mathfrak{m}^{(1,1)} \oplus \mathfrak{m}^{(0,2)}$ is a parabolic subalgebra of $\mathfrak{m}_{\mathbf{C}}$. From the description (2.2) of $\mathfrak{s p}_{\mathbf{C}}$, we see that $\mathfrak{m}^{(1,1)}$ consists of degree-preserving operators on $\mathscr{P}$, and $\mathfrak{m}^{(0,2)}$ consists of degree-decreasing operators.

Fact 3. One has

$$
\mathfrak{m}_{\mathbf{C}}=\left(\mathfrak{m}^{(1,1)} \oplus \mathfrak{m}^{(0,2)}\right)+\mathfrak{g}_{\mathbf{C}}, \quad \mathfrak{m}_{\mathbf{C}}^{\prime}=\left(\mathfrak{m}^{\prime(1,1)} \oplus \mathfrak{m}^{\prime(0,2)}\right)+\mathfrak{g}_{\mathbf{C}}^{\prime} .
$$

Actually, somewhat more precisely, one has

$$
\begin{aligned}
\mathfrak{m}^{(2,0)} \oplus \mathfrak{m}^{(0,2)}=\mathfrak{p}_{\mathbf{C}} \oplus \mathfrak{m}^{(0,2)}=\mathfrak{m}^{(2,0)} \oplus \mathfrak{p}_{\mathbf{C}}, \\
\mathfrak{m}^{\prime(2,0)} \oplus \mathfrak{m}^{\prime(0,2)}=\mathfrak{p}_{\mathbf{C}}^{\prime} \oplus \mathfrak{m}^{\prime(0,2)}=\mathfrak{m}^{\prime(2,0)} \oplus \mathfrak{p}_{\mathbf{C}}^{\prime} .
\end{aligned}
$$


Here $\mathfrak{p}$ and $\mathfrak{p}^{\prime}$ are the -1 eigenspaces for the Cartan involutions of $\mathfrak{g}$ and $\mathfrak{g}^{\prime}$, as in (2.4).

Example. It is less straightforward to illustrate Fact 3 than the first two facts, since it depends on knowing something about how $\mathfrak{g}$ sits in $\mathfrak{m}$, or $\mathfrak{g}^{\prime}$ in $\mathfrak{m}^{\prime}$. However, consider again the pair $\left(\mathfrak{o}_{p, q}, \mathfrak{s p}_{2 n}\right)$. Then as we have noted, $\mathfrak{m}^{\prime}=$ $\mathfrak{s p}_{2 n} \times \mathfrak{s p}_{2 n}$. Let $p_{i}, i=1,2$, be a projection of $\mathfrak{m}^{\prime}$ onto its $i$ th factor. It is not hard to see that $p_{i}$ is an isomorphism from $\mathfrak{g}^{\prime}=\mathfrak{s p}_{2 n}$ to each of the two factors. However, one of the $p_{i}$ is "holomorphic" and the other is "antiholomorphic" in the following sense:

$$
\begin{aligned}
& \left.p_{2}\left(p_{1}^{-1}\left(\mathfrak{m}^{\prime(2,0)}\right) \cap \mathfrak{g}_{\mathbf{C}}^{\prime}\right)\right) \subseteq \mathfrak{m}^{\prime(0,2)}, \\
& \left.p_{2}\left(p_{1}^{-1}\left(\mathfrak{m}^{\prime(0,2)}\right) \cap \mathfrak{g}_{\mathbf{C}}^{\prime}\right)\right) \subseteq \mathfrak{m}^{\prime(2,0)} .
\end{aligned}
$$

From these relations we can see that $\mathfrak{g}^{\prime} \cap \mathfrak{m}^{\prime(2,0)}=\{0\}=\mathfrak{g}^{\prime} \cap \mathfrak{m}^{(0,2)}$. The decompositions (3.5) then follow from this by dimension counting. The relation between $\mathfrak{g}=\mathfrak{o}_{p, q}$ and $\mathfrak{m}=\mathfrak{u}_{p, q}$ is similar.

These are the facts we need to describe the structure of $\mathscr{P}$ as a $\mathfrak{g} \times \mathfrak{g}^{\prime}$ module. We will also use the known structure for a dual pair $\left(L, L^{\prime}\right)$ for which $L$ is compact [H1]. Let us recall the essential aspects of these results. We assume again that $L \subseteq \mathrm{U}$, where as before $\mathrm{U}$ is the maximal compact of $\mathrm{Sp}$ used to define the Cartan decomposition (2.1), and in terms of which the Fock model

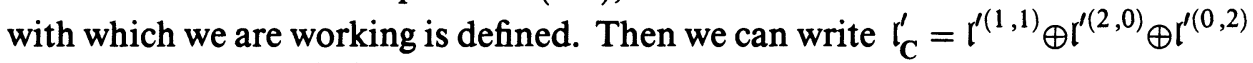
with $\mathfrak{r}^{\prime(i, j)}=\mathfrak{l}_{\mathbf{C}} \cap \mathfrak{s p}^{(i, j)}$ as usual. We set

$$
\mathscr{H}(L)=\left\{P \in \mathscr{P}: \ell(P)=0 \text { for all } \ell \in \mathfrak{r}^{(0,2)}\right\} .
$$

Consider the isotypic decomposition of $\mathscr{P}$ as an $L$-module:

$$
\mathscr{P}=\sum_{\sigma \in \mathscr{R}(L, \omega)} \mathscr{I}_{\sigma} .
$$

Here any $L$-invariant and irreducible subspace of $\mathscr{I}_{\sigma}$ defines a representation in the isomorphism class of $\sigma$. Since $\omega(L)$ preserves the degree of polynomials, the spaces $\mathscr{I}_{\sigma}$ are the sums of their homogeneous components. Let $\operatorname{deg}(\sigma)$ be the smallest possible degree of nonzero polynomials occurring in $\mathscr{F}_{\sigma}$.

Since $L$ commutes with $\mathfrak{l}^{\prime}$, in particular with $\mathfrak{l}^{\prime(0,2)}$, it is clear that $\mathscr{H}(L)$ is invariant under $L$. Thus,

$$
\mathscr{H}(L)=\sum_{\sigma} \mathscr{H}(L) \cap \mathscr{I}_{\sigma}
$$

Write, for $\sigma \in \mathscr{R}(L, \omega)$,

$$
\mathscr{H}(L)_{\sigma}=\mathscr{H}(L) \cap \mathscr{I}_{\sigma} .
$$

Since $\mathfrak{l}^{\prime}$ commutes with $L$, it is clear that $\mathscr{F}_{\sigma}$ is invariant under $\mathfrak{l}^{\prime}$, so that $\mathscr{I}_{\sigma}$ is actually an $L \times \mathfrak{l}^{\prime}$ module. In particular, $\mathscr{F}_{\sigma}$ is invariant under $\mathfrak{l}^{\prime(0,2)}$. 
Since the operators in $\mathfrak{l}^{\prime(0,2)}$ reduce degree, the polynomials of the smallest degree in $\mathscr{I}_{\sigma}$ must be in $\mathscr{H}(L)_{\sigma}$. Thus, if $\mathscr{J}_{\sigma} \neq\{0\}$, then also $\mathscr{H}(L)_{\sigma} \neq\{0\}$.

Since $\mathfrak{l}^{\prime(1,1)}$ normalizes $\mathfrak{l}^{\prime(0,2)}$, we see that $\mathscr{H}(L)$ will be invariant under $\mathfrak{l}^{(1,1)}$. Then also each space $\mathscr{H}(L)_{\sigma}$ will be invariant under $\mathfrak{l}^{(1,1)}$.

From Classical Invariant Theory, one may derive the following conclusions, as explained in $[\mathrm{H} 1]$.

(a) The joint action of $L \times \mathfrak{r}^{\prime}$ on $\mathscr{F}_{\sigma}$ is irreducible for each $\sigma \in \mathscr{R}(L, \omega)$. (3.9)

(b) The space $\mathscr{H}(L)_{\sigma}$ consists precisely of the space of polynomials of lowest degree $\operatorname{deg}(\sigma)$ in $\mathscr{I}_{\sigma}$.

(c) One has $\mathscr{I}_{\sigma}=\mathscr{U}\left(\mathfrak{l}^{\prime(2,0)}\right) \cdot \mathscr{H}(L)_{\sigma}$, where $\mathscr{U}\left(\mathfrak{l}^{\prime(2,0)}\right)$ is the universal enveloping algebra of $\mathfrak{l}^{\prime(2,0)}$.

(d) The group $L$ and the Lie algebra $\mathfrak{r}^{(1,1)}$ generate mutual commutants on $\mathscr{H}(L)$. Equivalently, each $\mathscr{H}(L)_{\sigma}$ is irreducible under the joint action of $L \times \mathfrak{r}^{\prime(1,1)}$, and if we write $\mathscr{H}(L)_{\sigma} \simeq \sigma \otimes \tau^{\prime}$ for $\tau^{\prime} \in \mathscr{R}\left(\mathfrak{l}^{\prime(1,1)}, \omega\right)$, then $\sigma$ determines $\tau^{\prime}$ and vice versa, so that $\sigma \rightarrow \tau^{\prime}$ is an injection from $\mathscr{R}(L, \omega)$ into $\mathscr{R}\left(\mathfrak{l}^{(1,1)}, \omega\right)$.

To first apply these facts, let $L$ be $K$ or $K^{\prime}$, the maximal compact subgroups of $G$ or $G^{\prime}$. (We will no longer distinguish between $K$ and $\widetilde{K}$, etc. This should not cause confusion.) Then $L^{\prime}$ will be $M^{\prime}$ or $M$, respectively. We may consider the harmonics $\mathscr{H}(K)$ and $\mathscr{H}\left(K^{\prime}\right)$. We will denote a typical element of $\mathscr{R}(K, \omega)$ by $\sigma$ and one of $\mathscr{R}\left(K^{\prime}, \omega\right)$ by $\sigma^{\prime}$. Keeping in mind that $\mathscr{H}(K)$ will be stabilized by $\mathfrak{m}^{\prime(1,1)} \oplus \mathfrak{m}^{(0,2)}$, we observe that combining statement $(3.9)(\mathrm{c})$ with Fact 3 ((3.4) and (3.5)) immediately yields the following result.

Proposition 3.1. The space $\mathscr{H}(K)$ generates $\mathscr{P}$ as a $\mathfrak{g}$ ' module, and similarly for $K^{\prime}$. That is,

$$
\mathscr{P}=\mathscr{U}(\mathfrak{g}) \cdot \mathscr{H}(K)=\mathscr{U}(\mathfrak{g}) \cdot \mathscr{H}\left(K^{\prime}\right),
$$

where $\mathscr{U}\left(\mathfrak{g}^{\prime}\right)$ is the universal enveloping algebra of $\mathfrak{g}^{\prime}$. Thus, for any $\sigma \in$ $\mathscr{R}(K, \omega)$ or $\sigma^{\prime} \in \mathscr{R}\left(K^{\prime}, \omega\right)$, one has

$$
\mathscr{I}_{\sigma}=\mathscr{U}\left(\mathfrak{g}^{\prime}\right) \cdot \mathscr{H}(K)_{\sigma}, \quad \mathscr{I}_{\sigma^{\prime}}=\mathscr{U}(\mathfrak{g}) \cdot \mathscr{H}\left(K^{\prime}\right)_{\sigma} .
$$

Here $\mathscr{I}_{\sigma}$ is the $\sigma$-isotypic subspace of $\mathscr{P}$, and likewise for $\mathscr{I}_{\sigma^{\prime}}$.

Consider the pair $\left(\mathfrak{m}_{0}^{(1,1)}, \mathfrak{m}_{0}^{\prime(1,1)}\right)$, which we know is a dual pair by Fact 2 . Let $\left(M^{(1,1)}, M^{\prime(1,1)}\right)$ be the associated pair of groups. As we observed, both $M^{(1,1)}$ and $M^{\prime(1,1)}$ are products of unitary groups. In particular, they are both compact. Thus, the statements (3.9) for this pair simply amount to the statement that if

$$
\mathscr{P}=\sum_{\tau \in \mathscr{R}\left(M^{(1,1)}, \omega\right)} \mathscr{J}_{\tau}=\sum_{\tau^{\prime} \in \mathscr{R}\left(M^{\prime(1,1)}, \omega\right)} \mathscr{J}_{\tau^{\prime}}
$$


are the isotypic decompositions of $\mathscr{P}$ for $M^{(1,1)}$ and for $M^{\prime(1,1)}$, then there is a bijection

$$
\begin{aligned}
\mathscr{R}\left(M^{(1,1)}, \omega\right) & \leftrightarrow \mathscr{R}\left(M^{\prime(1,1)}, \omega\right), \\
\tau & \leftrightarrow \tau^{\prime}
\end{aligned}
$$

such that

$$
\mathscr{J}_{\tau}=\mathscr{J}_{\tau^{\prime}}
$$

We will call this common isotypic subspace $\mathscr{J}_{\tau, \tau^{\prime}}$. Thus, the $\mathscr{J}_{\tau, \tau^{\prime}}$ are finite dimensional and irreducible for the action of $M^{(1,1)} \times M^{\prime(1,1)}$. The representation of $M^{(1,1)} \times M^{\prime(1,1)}$ on $\mathscr{J}_{\tau, \tau^{\prime}}$ is, of course, $\tau \otimes \tau^{\prime}$.

Since $K \subseteq M^{(1,1)}$, the space $\mathscr{J}_{\tau, \tau^{\prime}}$ is invariant under $K$. The action of $K \times M^{\prime(1,1)}$ on $\mathscr{J}_{\tau, \tau^{\prime}}$ is of course $(\tau \mid K) \otimes \tau^{\prime}$. Thus, if one has $\tau \mid K \simeq \sum_{\sigma} a_{\tau, \sigma} \sigma$, then

$$
\operatorname{dim}\left(\mathscr{I}_{\sigma} \cap \mathscr{J}_{\tau, \tau^{\prime}}\right)=a_{\tau, \sigma} \operatorname{dim} \sigma \operatorname{dim} \tau^{\prime}
$$

Note that

$$
\mathscr{J}_{\tau, \tau^{\prime}}=\sum_{\sigma} \mathscr{J}_{\tau, \tau^{\prime}} \cap \mathscr{I}_{\sigma}
$$

Consider the space $\mathscr{H}(K) \cap \mathscr{J}_{\tau, \tau^{\prime}}$. Since $\mathscr{H}(K)$ is invariant under $M^{\prime(1,1)}$ and $\mathscr{J}_{\tau, \tau^{\prime}}$ is isotypic for $M^{\prime(1,1)}$, we have

$$
\mathscr{H}(K)=\sum_{\tau^{\prime}} \mathscr{H}(K) \cap \mathscr{J}_{\tau, \tau^{\prime}}
$$

Observe that $\mathscr{J}_{\tau, \tau^{\prime}}$ will consist entirely of polynomials homogeneous of degree $\operatorname{deg} \tau=\operatorname{deg} \tau^{\prime}$. Thus, we can have $\mathscr{I}_{\sigma} \cap \mathscr{J}_{\tau, \tau^{\prime}} \neq\{0\}$ only if $\operatorname{deg} \sigma \leq \operatorname{deg} \tau$. From statement (3.9)(b), we see that if $\mathscr{J}_{\sigma} \cap \mathscr{J}_{\tau, \tau^{\prime}} \neq\{0\}$, then $\mathscr{H}(K)_{\sigma} \cap \mathscr{J}_{\tau, \tau^{\prime}} \neq\{0\}$ if and only if $\operatorname{deg} \sigma=\operatorname{deg} \tau$. Furthermore, since $\mathscr{J}_{\tau, \tau^{\prime}}$ is isotypic for $M^{\prime(1,1)}$, we see from statement $(3.9)(\mathrm{d})$ that $\mathscr{H}(K) \cap \mathscr{J}_{\tau, \tau^{\prime}}$ must be irreducible. Thus, we have

Lemma 3.2. Either $\mathscr{H}(K) \cap \mathscr{J}_{\tau, \tau^{\prime}}=0$ or there is a unique $\sigma \in \mathscr{R}(K, \omega)$ such that $\operatorname{deg} \sigma=\operatorname{deg} \tau^{\prime}$ and one has the $K$-module decomposition

$$
\mathscr{J}_{\tau, \tau^{\prime}}=\mathscr{H}(K)_{\sigma} \oplus \sum_{\operatorname{deg} \tilde{\sigma}<\operatorname{deg} \tau^{\prime}} \mathscr{I}_{\tilde{\sigma}} \cap \mathscr{J}_{\tau, \tau^{\prime}} .
$$

The analogous statement holds for $K^{\prime}$. 
If we combine the decomposition (3.14) with the analogous one for $K^{\prime}$, we find

Lemma 3.3. If $\mathscr{J}_{\tau, \tau^{\prime}} \cap \mathscr{H}(K) \neq\{0\} \neq \mathscr{I}_{\tau, \tau^{\prime}} \cap \mathscr{H}\left(K^{\prime}\right)$, then under the action of $K \times K^{\prime}$ the space $\mathscr{J}_{\tau, \tau^{\prime}}$ has the decomposition

$$
\begin{gathered}
\mathscr{J}_{\tau, \tau^{\prime}}=\mathscr{H}(K)_{\sigma} \cap \mathscr{H}\left(K^{\prime}\right)_{\sigma^{\prime}} \oplus \sum_{\operatorname{deg} \tilde{\sigma}<\operatorname{deg} \tau^{\prime}} \mathscr{I}_{\tilde{\sigma}} \cap \mathscr{H}\left(K^{\prime}\right)_{\sigma^{\prime}} \\
\oplus \sum_{\operatorname{deg} \tilde{\sigma}^{\prime}<\operatorname{deg} \tau} \mathscr{H}(K)_{\sigma} \cap \mathscr{I}_{\tilde{\sigma}^{\prime}} \\
\oplus \sum_{\operatorname{deg} \tilde{\sigma}, \operatorname{deg} \tilde{\sigma}^{\prime}<\operatorname{deg} \tau} \mathscr{I}_{\tilde{\sigma}} \cap \mathscr{I}_{\tilde{\sigma}^{\prime}} \cap \mathscr{J}_{\tau, \tau^{\prime}} .
\end{gathered}
$$

Moreover, one has $\operatorname{deg} \sigma=\operatorname{deg} \sigma^{\prime}=\operatorname{deg} \tau=\operatorname{deg} \tau^{\prime}$ and $\sigma$ and $\sigma^{\prime}$ are uniquely determined by $\tau$ and $\tau^{\prime}$, and vice versa. Also the space $\mathscr{H}(K)_{\sigma} \cap \mathscr{H}(K)_{\sigma^{\prime}}$ is irreducible under the action of $K \times K^{\prime}$. Finally, $\sigma$ and $\sigma^{\prime}$ determine each other, so that $K$ and $K^{\prime}$ generate mutual commutants on $\mathscr{H}(K) \cap \mathscr{H}\left(K^{\prime}\right)$.

Finally, we have

Proposition 3.4. The space $\mathscr{H}(K) \cap \mathscr{H}\left(K^{\prime}\right)$ generates $\mathscr{P}$ as a $\mathfrak{g} \times \mathfrak{g}^{\prime}$ module. That is, we have

$$
\mathscr{P}=\mathscr{U}(\mathfrak{g}) \mathscr{U}\left(\mathfrak{g}^{\prime}\right)\left(\mathscr{H}(K) \cap \mathscr{H}\left(K^{\prime}\right)\right) .
$$

Proof. We argue by induction on $\operatorname{deg} \tau=\operatorname{deg} \tau^{\prime}$ that $\mathscr{J}_{\tau, \tau^{\prime}}$ is contained in the right-hand side of (3.16). For a given $d$, let $\mathscr{P}^{(d)}$ denote the space of polynomials of degree at most $d$, and let $\mathscr{Y}^{(d)}$ denote the $\mathfrak{g} \times \mathfrak{g}^{\prime}$ submodule of $\mathscr{P}$ generated by $\mathscr{P}^{(d)}$. We may assume inductively that $\mathscr{Y}^{(d)}$ is, in fact, generated by $\mathscr{P}^{(d)} \cap \mathscr{H}(K) \cap \mathscr{H}\left(K^{\prime}\right)$. This is automatically true for $d=0$ since the constants are both $K$ and $K^{\prime}$ harmonic.

Consider the decomposition (3.15) of $\mathscr{J}_{\tau, \tau^{\prime}}$. According to Proposition 3.1 and statement (3.9)(b), we have

$$
\mathscr{J}_{\tilde{\sigma}} \cap \mathscr{H}\left(K^{\prime}\right)_{\sigma^{\prime}} \subseteq \mathscr{U}\left(\mathfrak{g}^{\prime}\right) \mathscr{H}(K)_{\tilde{\sigma}} \subseteq \mathscr{Y}^{(d)},
$$

where $d=\operatorname{deg} \tilde{\sigma}$. A similar fact holds for $\mathscr{H}(K)_{\sigma} \cap \mathscr{I}_{\tilde{\sigma}}$ and for $\mathscr{I}_{\tilde{\sigma}} \cap \mathscr{J}_{\tilde{\sigma}^{\prime}}$. This leaves only $\mathscr{H}(K)_{\sigma} \cap \mathscr{H}\left(K^{\prime}\right)_{\sigma^{\prime}}$ to account for, but the result is automatic here. The proposition follows.

\section{Proof of Theorem 2.1}

Let $\mathscr{N} \subseteq \mathscr{P}$ be an arbitrary subspace of $\mathscr{P}$ which is invariant under $\mathfrak{g}, \mathfrak{g}^{\prime}$, and $K \cdot K^{\prime}$, and consider the $\left(\mathfrak{g} \times \mathfrak{g}^{\prime}, K \cdot K^{\prime}\right)$ quotient module $\mathscr{P} / \mathscr{N}$. Recall that $\mathscr{P}^{(d)}$ denotes the space of polynomials of degree at most $d$, and $\mathscr{Y}^{(d)}$ the $\left(\mathfrak{g} \times \mathfrak{g}^{\prime}, K \cdot K^{\prime}\right)$ module generated by $\mathscr{P}^{(d)}$. Let $\delta(\mathscr{N})$ be the largest integer $d$ such that $\mathscr{P}^{(d)}$ (hence $\mathscr{Y}^{(d)}$ ) is contained in $\mathscr{N}$. 
Lemma 4.1. (a) The number $\delta(\mathscr{N})$ is determined solely by the structure of $\mathscr{P} / \mathscr{N}$ as a $K$ module, or as a $K^{\prime}$ module. Specifically, one has

$$
\begin{aligned}
1+\delta(\mathscr{N}) & =\min \{\operatorname{deg} \sigma: \sigma \in \mathscr{R}(K, \mathscr{P} / \mathscr{N})\} \\
& =\min \left\{\operatorname{deg} \sigma^{\prime}: \sigma^{\prime} \in \mathscr{R}\left(K^{\prime}, \mathscr{P} / \mathscr{N}\right)\right\},
\end{aligned}
$$

where $\mathscr{R}(K, \mathscr{P} / \mathscr{N})$ is the set of irreducible representations of $K$ occurring in $\mathscr{P} / \mathscr{N}$, and likewise for $\mathscr{R}\left(K^{\prime}, \mathscr{P} / \mathscr{N}\right)$.

(b) If $\sigma \in \mathscr{R}(K, \mathscr{P} / \mathscr{N})$, then one of the finite number of $\sigma^{\prime} \in \mathscr{R}\left(K^{\prime}, \omega\right)$ such that $\mathscr{H}(K)_{\sigma} \cap \mathscr{I}_{\sigma^{\prime}} \neq\{0\}$ occurs in $\mathscr{P} / \mathscr{N}$.

(c) If $\sigma \in \mathscr{R}(K, \mathscr{P} / \mathscr{N})$ and $\operatorname{deg} \sigma=\delta(\mathscr{N})+1$, then $\mathscr{H}(K)_{\sigma} \cap \mathscr{H}\left(K^{\prime}\right) \neq$ $\{0\}$. If $\mathscr{H}(K)_{\sigma} \cap \mathscr{H}\left(K^{\prime}\right) \simeq \sigma \otimes \sigma^{\prime}$, then $\sigma^{\prime} \in \mathscr{R}\left(K^{\prime}, \mathscr{P} / \mathscr{N}\right)$, and no other representation of $K^{\prime}$ occurring in $\mathscr{H}(K)_{\sigma}$ occurs in $\mathscr{P} / \mathscr{N}$.

Proof. Consider $\sigma$ in $\mathscr{R}(K, \mathscr{P} / \mathscr{N})$. Let $(\mathscr{P} / \mathscr{N})_{\sigma}$ denote the $\sigma$-isotypic component of $\mathscr{P} / \mathscr{N}$. Then $(\mathscr{P} / \mathscr{N})_{\sigma}$ is a $K \times\left(\mathfrak{g}^{\prime}, K^{\prime}\right)$ module and is a quotient of $\mathscr{I}_{\sigma}$, the $\sigma$-isotypic component of $\mathscr{P}$. Precisely, one has

$$
(\mathscr{P} / \mathscr{N})_{\sigma}=\mathscr{I}_{\sigma} /\left(\mathscr{N} \cap \mathscr{I}_{\sigma}\right) \text {. }
$$

From Proposition 3.1, especially equation (3.10)(b), we see that $\mathscr{H}(K)_{\sigma} \nsubseteq \mathscr{N}$, so that the image of $\mathscr{H}(K)_{\sigma}$ in $\mathscr{P} / \mathscr{N}$ is nonzero. Hence, $\delta(\mathscr{N})<\operatorname{deg} \sigma$. Thus, the left-hand side of equation (4.1) is not greater than the right-hand side. The reverse inequality is trivial. Hence, (a) of the lemma follows.

From the observation that if $\sigma \in \mathscr{R}(K, \mathscr{P} / \mathscr{N})$, then $\mathscr{H}(K)_{\sigma}$ has nonzero image in $\mathscr{P} / \mathscr{N}$, part (b) of the lemma is immediate. To prove part (c), suppose $\mathscr{H}(K)_{\sigma} \cap \mathscr{I}_{\sigma^{\prime}}$ has nonzero image in $\mathscr{P} / \mathscr{N}$. Then equation (4.1) implies $\operatorname{deg} \sigma^{\prime} \geq \delta(\mathscr{N})+1=\operatorname{deg} \sigma$. Hence $\mathscr{H}(K)_{\sigma} \cap \mathscr{J}_{\sigma^{\prime}}=\mathscr{H}(K)_{\sigma} \cap \mathscr{H}\left(K^{\prime}\right)_{\sigma^{\prime}}$. This finishes the lemma.

Now fix $\rho \in \mathscr{R}(\mathfrak{g}, K, \omega)$ and set $\mathscr{N}=\mathscr{N}_{\rho}$ as in (2.4). Pick a $\sigma \in$ $\mathscr{R}\left(K, \mathscr{P} / \mathscr{N}_{\rho}\right)$ such that $(4.1)$ holds. Let $\sigma^{\prime}$ be the associated representation of $K^{\prime}$, as in Lemma 4.1(c). Write $\mathscr{H}(K)_{\sigma} \cap \mathscr{H}\left(K^{\prime}\right)_{\sigma^{\prime}}=\mathscr{H}_{\sigma, \sigma^{\prime}}$. According to the proof of Lemma 4.1, we know that $\mathscr{H}_{\sigma, \sigma^{\prime}}$ generates $\left(\mathscr{P} / \mathscr{N}_{\rho}\right)_{\sigma}$, the $\sigma$-isotypic component $\mathscr{P} / \mathscr{N}_{\rho}$, as a $\mathfrak{g}^{\prime}$ module. (2.4) tells us that

$$
\left(\mathscr{P} / \mathscr{N}_{\rho}\right)_{\sigma} \simeq \rho_{\sigma} \otimes \rho_{1}^{\prime} \text {. }
$$

It follows that $\rho_{1}^{\prime}$ is generated as a $\mathfrak{g}^{\prime}$ module by $\left(\rho_{1}^{\prime}\right)_{\sigma^{\prime}}$, its $\sigma^{\prime}$-isotypic component for $K^{\prime}$. Furthermore, since $\rho$ is irreducible, it follows that $\mathscr{H}_{\sigma, \sigma^{\prime}}$ generates $\mathscr{P} / \mathscr{N}_{\rho}$ as a $\mathfrak{g} \times \mathfrak{g}^{\prime}$ module.

Let $\tau$ and $\tau^{\prime}$ be the representations of $M^{(1,1)}$ and $M^{\prime(1,1)}$ corresponding to $\sigma^{\prime}$ and $\sigma$, respectively, via statement (3.9)(d). We have seen (Lemma 3.3) that $\mathscr{H}_{\sigma, \sigma^{\prime}}=\mathscr{J}_{\tau, \tau^{\prime}} \cap \mathscr{H}(K) \cap \mathscr{H}\left(K^{\prime}\right)$. Here $\mathscr{J}_{\tau, \tau^{\prime}}$ is as in (3.13). Let $\mathscr{Y}^{\tau, \tau^{\prime}}$ be the $\mathfrak{g} \times \mathfrak{g}^{\prime}$ module generated by $\mathscr{J}_{\tau, \tau^{\prime}}$. Set $d=\operatorname{deg} \sigma-1=\delta\left(\mathcal{N}_{\rho}\right)$, and define

$$
\mathscr{Z}^{\tau, \tau^{\prime}}=\left(\mathscr{Y}^{\tau, \tau^{\prime}}+\mathscr{Y}^{(d)}\right) / \mathscr{Y}^{(d)} \text {. }
$$


We have just seen that $\mathscr{P} / \mathscr{N}_{\rho}$ is, in fact, a quotient of $\mathscr{Z}^{\tau, \tau^{\prime}}$. This leads us to study some features of $\mathscr{Z}^{\tau, \tau^{\prime}}$.

We recall some well-known general facts about $(\mathfrak{g}, K)$ modules [D]. Let $\mathscr{M}$ be a $(\mathfrak{g}, K)$ module, and let $\mu: \mathscr{U}(\mathfrak{g}) \rightarrow \operatorname{End}(\mathscr{M})$ be the associated action of $\mathscr{U}(\mathfrak{g})$. Let $\mathscr{U}(\mathfrak{g})^{K}$ be the centralizer of $K$, and let $E_{\sigma}$ be the projection of $\mathscr{M}$ on $\mathscr{M}_{\sigma}$. Then one has an isomorphism

$$
\text { End } \sigma \cdot E_{\sigma} \mu(\mathscr{U}(\mathfrak{g})) E_{\sigma} \simeq \text { End } \sigma \otimes E_{\sigma} \mu\left(\mathscr{U}(\mathfrak{g})^{K}\right)
$$

Return to consideration of $\mathscr{Z}^{\tau, \tau^{\prime}}$. Abbreviate $\mathscr{Z}^{\tau, \tau^{\prime}}=\mathscr{Z}$. We wish to consider the $\sigma$-isotypic component for $\sigma, \mathscr{Z}_{\sigma}$; the $\sigma^{\prime}$-isotypic component for $K^{\prime}$, $\mathscr{Z}_{\sigma^{\prime}}$; and their intersection $\mathscr{Z}_{\sigma} \cap \mathscr{Z}_{\sigma^{\prime}}=\mathscr{Z}_{\sigma, \sigma^{\prime}}$, which is the $\sigma \otimes \sigma^{\prime}$-isotypic component for $K \cdot K^{\prime}$. Of course, $\mathscr{Z}_{\sigma}$ is a $\left(\mathfrak{g}^{\prime}, K^{\prime}\right)$-module, so we may consider its $K^{\prime}$-isotypic components, and similarly for $\mathscr{Z}_{\sigma^{\prime}}$. We have the obvious equalities

$$
\mathscr{Z}_{\sigma, \sigma^{\prime}}=\left(\mathscr{Z}_{\sigma}\right)_{\sigma^{\prime}}=\left(\mathscr{Z}_{\sigma^{\prime}}\right)_{\sigma} \text {. }
$$

We abuse notation to let $\mathscr{H}_{\sigma, \sigma^{\prime}}$ also denote the image of $\mathscr{H}_{\sigma, \sigma^{\prime}}$ in $\mathscr{Z}$. Combining $(3.9)(\mathrm{c})$ with Lemma 4.1(c), we have

$$
\mathscr{Z}_{\sigma}=\mathscr{U}\left(\mathfrak{g}^{\prime}\right) \cdot \mathscr{H}_{\sigma, \sigma^{\prime}}, \quad \mathscr{Z}_{\sigma^{\prime}}=\mathscr{U}(\mathfrak{g}) \cdot \mathscr{H}_{\sigma, \sigma^{\prime}} .
$$

Combining (4.3), (4.4), and (4.5) yields

$$
\mathscr{Z}_{\sigma, \sigma^{\prime}}=\mathscr{U}(\mathfrak{g})^{K} \cdot \mathscr{H}_{\sigma, \sigma^{\prime}}=\mathscr{U}\left(\mathfrak{g}^{\prime}\right)^{K} \cdot \mathscr{H}_{\sigma, \sigma^{\prime}} .
$$

Since $\mathscr{H}_{\sigma, \sigma^{\prime}}$ is irreducible under $K \cdot K^{\prime}$, we have a canonical isomorphism

$$
\mathscr{Z}_{\sigma, \sigma^{\prime}} \simeq \mathscr{H}_{\sigma, \sigma^{\prime}} \otimes \operatorname{Hom}_{K \cdot K^{\prime}}\left(\mathscr{H}_{\sigma, \sigma^{\prime}}, \mathscr{Z}\right)
$$

defined by the map

$$
T \otimes h \rightarrow T(h), \quad h \in \mathscr{H}_{\sigma, \sigma^{\prime}}, T \in \operatorname{Hom}_{K \cdot K^{\prime}}\left(\mathscr{H}_{\sigma, \sigma^{\prime}}, \mathscr{Z}\right) .
$$

It is clear that under the isomorphism (4.7), the algebras $\mathscr{U}(\mathfrak{g})^{K}$ and $\mathscr{U}\left(\mathfrak{g}^{\prime}\right)^{K^{\prime}}$ act on the second factor $\operatorname{Hom}_{K \cdot K^{\prime}}\left(\mathscr{H}_{\sigma, \sigma^{\prime}}, \mathscr{Z}\right)=Z_{\sigma, \sigma^{\prime}}$. Let $e_{0}$ be the distinguished vector in $Z_{\sigma, \sigma^{\prime}}$ defined by the identity map on $\mathscr{H}_{\sigma, \sigma^{\prime}}$. Then (4.6) may be restated as

$$
Z_{\sigma, \sigma^{\prime}}=\mathscr{U}(\mathfrak{g})^{K}\left(e_{0}\right)=\mathscr{U}\left(\mathfrak{g}^{\prime}\right)^{K^{\prime}}\left(e_{0}\right)
$$

(4.8) is the key to proving Theorem 2.1. We note a very simple lemma.

Lemma 4.2. Let $A$ and $B$ be algebras which act on a vector space $V$. Suppose $A$ and $B$ commute with one another. Suppose also there is a vector $v_{0} \in V$ which is a simultaneous cyclic vector for $A$ and for $B$; that is,

$$
A\left(v_{0}\right)=V=B\left(v_{0}\right) \text {. }
$$

Then $B$ is the full commutant of $A$ in End $V$ and vice versa. 
Proof. Suppose $T \in$ End $V$ commutes with $A$. Since $B\left(v_{0}\right)=V$, we can find $b_{T} \in B$ such that $T\left(v_{0}\right)=b_{T}\left(v_{0}\right)$. We will show $T=b_{T}$. Consider $v \in V$. Since $A\left(v_{0}\right)=V$, we can find $a \in A$ such that $a\left(v_{0}\right)=v$. We compute

$$
T(v)=T a\left(v_{0}\right)=a T\left(v_{0}\right)=a b_{T}\left(v_{0}\right)=b_{T} a\left(v_{0}\right)=b_{T}(v) .
$$

This proves the lemma.

Now we prove Theorem 2.1. Suppose $\mathscr{P} / \mathscr{N}_{\rho}$ allowed two irreducible quotients, isomorphic respectively to $\rho \otimes \rho^{\prime}$ and $\rho \otimes \rho^{\prime \prime}$ as $\left(\mathfrak{g} \times \mathfrak{g}^{\prime}, K \cdot K^{\prime}\right)$ modules. Each of these modules, and their direct sum, will be a quotient of $\mathscr{Z}_{\tau, \tau^{\prime}}$. Consider the $\sigma \otimes \sigma^{\prime}$-isotypic component for $K \cdot K^{\prime}$ in $\rho \otimes\left(\rho^{\prime} \oplus \rho^{\prime \prime}\right)$ and consider the action of $\mathscr{U}(\mathfrak{g})^{K}$ and of $\mathscr{U}\left(\mathfrak{g}^{\prime}\right)^{K^{\prime}}$ on $\operatorname{Hom}_{K \cdot K^{\prime}}\left(\mathscr{H}_{\sigma, \sigma^{\prime}}, \rho \otimes\left(\rho^{\prime} \oplus \rho^{\prime \prime}\right)\right)=X$. Let $e_{0}$ be the image in this space of the vector $e_{0}$ in (4.8). Since we are dealing with a quotient of $\mathscr{Z}_{\tau, \tau^{\prime}}$, we see this $e_{0}$ is again a cyclic vector for the action of $\mathscr{U}(\mathfrak{g})^{K}$ and of $\mathscr{U}\left(\mathfrak{g}^{\prime}\right)^{K^{\prime}}$ on $X$. Applying Lemma 4.2, we find the two algebras must be mutual commutants in $\operatorname{End}(X)$. On the other hand, projection of $\rho \otimes\left(\rho^{\prime} \oplus \rho^{\prime \prime}\right)$ on the first summand $\rho \otimes \rho^{\prime}$ will induce on $X$ a transformation which commutes with $\mathscr{U}\left(\mathfrak{g}^{\prime}\right)^{K^{\prime}}$ but does not belong to $\mathscr{U}(\mathfrak{g})^{K}$. This contradiction shows it is impossible to have two distinct irreducible quotients of $\mathscr{P} / \mathscr{N}_{\rho}$. This is the second and main statement of Theorem 2.1.

We have already seen that $\rho_{1}^{\prime}$ is generated by the $\left(\mathfrak{g}^{\prime}, K^{\prime}\right)$ module $\mathscr{H}_{\sigma, \sigma^{\prime}}$. Since $\rho$ is irreducible and admissible, it is quasisimple, so that $\mathscr{Z} \mathscr{U}(\mathfrak{g})^{K}$ acts by scalars on $\rho$. It follows by the remark in $[\mathrm{H} 1, \S 5 \mathrm{a}]$ that $\rho_{1}^{\prime}$ is also quasisimple; in fact, $\mathscr{Z} \mathscr{U}\left(\mathfrak{g}^{\prime}\right)^{K^{\prime}}$ acts by scalars in a way determined by the action of $\mathscr{Z} \mathscr{U}(\mathfrak{g})^{K}$. Hence, $\rho_{1}^{\prime}$ is finitely generated and quasisimple. It then follows from a general result [D] that $\rho_{1}^{\prime}$ is also admissible. This finishes Theorem 2.1.

\section{StRucture of NONCOMPACT DUAL PAIRS}

In this section, we verify Facts 1,2 , and 3 about dual pairs used in $\S 3$. There should be a very slick way of doing this, but I unfortunately do not have it. We must, at least to some extent, resort to the classification of reductive dual pairs, cf. [H2, MVW]. Similarities between several classes of pairs permit one to avoid excessive tedium.

It is clearly enough the prove the facts for irreducible dual pairs, of which there are seven families. As described in [H2], irreducible dual pairs may be divided into two types, type I and type II. Those of type II correspond to division algebras, of which there are three containing $\mathbf{R}$, namely, $\mathbf{R}$ itself, the complex numbers $\mathbf{C}$, and the quaternions $\mathbf{H}$. To each of these corresponds a family of irreducible pairs of type II. A list of them follows. 

Division algebra
Dual pair family
(i)
$\mathbf{R}$
$\left(\mathrm{GL}_{m}(\mathbf{R}), \mathrm{GL}_{n}(\mathbf{R})\right) \subseteq \mathrm{Sp}_{2 n m}(\mathbf{R})$
(ii)
(iii)
C
$\left(\mathrm{GL}_{m}(\mathbf{C}), \mathrm{GL}_{n}(\mathbf{C})\right) \subseteq \mathrm{Sp}_{4 m n}(\mathbf{R})$
$\left(\mathrm{GL}_{m}(\mathbf{H}), \mathrm{GL}_{n}(\mathbf{H})\right) \subseteq \mathrm{Sp}_{8 m n}(\mathbf{R})$

Irreducible pairs of type I are associated to division algebras with involution. There are four of these containing $\mathbf{R}$, namely, $\mathbf{R}$ with the trivial involution, $\mathbf{C}$ with the trivial involution, $\mathbf{C}$ with complex conjugation, and $\mathbf{H}$ with quaternionic conjugation. Again we list the families of dual pairs.

\begin{tabular}{rcc} 
& $\begin{array}{c}\text { Division algebra } \\
\text { with involution }\end{array}$ & \multicolumn{1}{c}{ Dual pair family } \\
(i) & $(\mathbf{R}, 1)$ & $\left(\mathrm{O}_{p, q}, \mathrm{Sp}_{2 n}(\mathbf{R})\right) \subseteq \mathrm{Sp}_{2(p+q) n}(\mathbf{R})$ \\
$($ ii) & $(\mathbf{C}, 1)$ & $\left(\mathrm{O}_{p}(\mathbf{C}), \mathrm{Sp}_{2 n}(\mathbf{C})\right) \subseteq \mathrm{Sp}_{4 p n}(\mathbf{R})$ \\
(iii) & $\left(\mathbf{C},{ }^{-}\right)$ & $\left(\mathrm{U}_{p, q}, \mathrm{U}_{r, s}\right) \subseteq \mathrm{Sp}_{2(p+q)(r+s)}(\mathbf{R})$ \\
(iv) & $\left(\mathbf{H},{ }^{-}\right)$ & $\left(\mathrm{Sp}_{p, q}, \mathrm{O}_{2 n}^{*}\right) \subseteq \mathrm{Sp}_{4 n(p+q)}(\mathbf{R})$
\end{tabular}

In (5.2), the notation for the groups are consistent with Helgason [Hl, Chapter IX], except our $\operatorname{Sp}_{2 n}(\mathbf{R})$ and $\operatorname{Sp}_{2 n}(\mathbf{C})$ are his $\operatorname{Sp}(n, \mathbf{R})$ and $\operatorname{Sp}(n, \mathbf{C})$. The in $\left(\mathbf{C},{ }^{-}\right)$and $\left(\mathbf{H},{ }^{-}\right)$indicates the appropriate conjugation.

Observe that in (5.2), except for the family (5.2)(ii), the first group in each pair is capable of being compact (if $p=0$ or $q=0$ ), and the second group has Hermitian structure. If the first member of the pair is noncompact, its maximal compact subgroup is a product of two groups of the same type. Thus, the maximal compact subgroup of $\mathrm{O}_{p, q}$ is $\mathrm{O}_{p, 0} \times \mathrm{O}_{0, q}$, and so forth. It is therefore clear that these groups belong to dual pairs, the second member of which is a product of two copies of the second member of the original pair. (Note that this pair is reducible.)

The maximal compact subgroup of the second member of the pair is a unitary group (or in the case of family (5.2)(iii), whose first and second members are not really distinguishable, a product of unitary groups). It belongs to a dual pair of type (5.2)(iii).

The other pairs can be checked similarly. We compile in (5.3) a table of the families of pairs, their maximal compact subgroups, and the pairs to which they belong. Here we use the notation $G, K, M, M^{(1,1)}$ as in $\S 3$.

(5.3) exhibits some regularities which are worth noting. First, as we observed, $\mathfrak{m}_{C}$ will always allow the decomposition (3.2), that is, it will be a graded subalgebra of $\mathfrak{s p}_{\mathbf{C}}$ with respect to the grading (2.2). In fact, it is the smallest graded subalgebra of $\mathfrak{s p}_{\mathbf{C}}$ containing $g$. This, in fact, is the content of Fact 3 . Second, the group $M^{(1,1)}$ is the intersection with the unitary subgroup (of the large Sp containing $\left(G, G^{\prime}\right)$ ) with the algebra (of operators on the symplectic space $W$ on which $\mathrm{Sp}$ is acting) generated by $K$. This makes it clear that $M^{(1,1)}$ and 

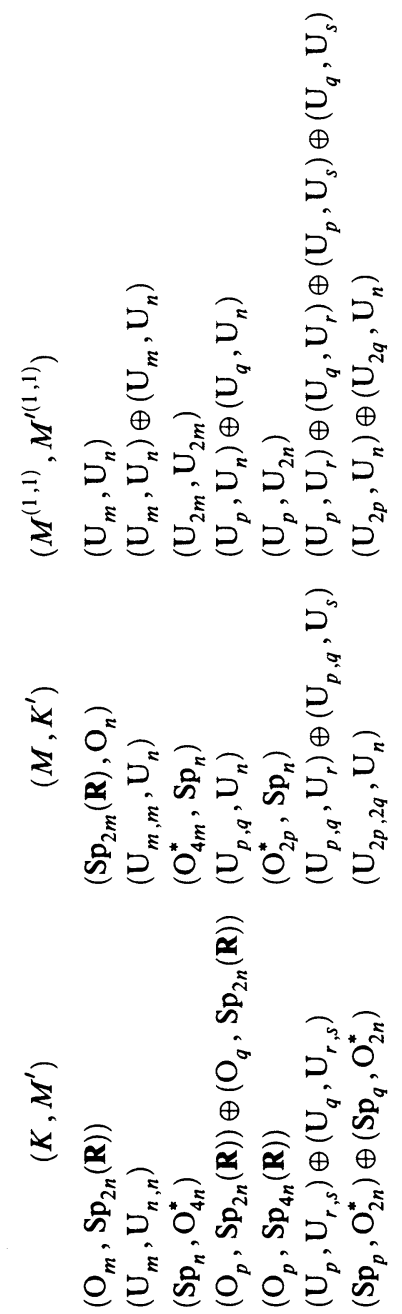

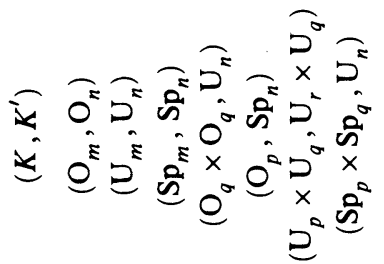

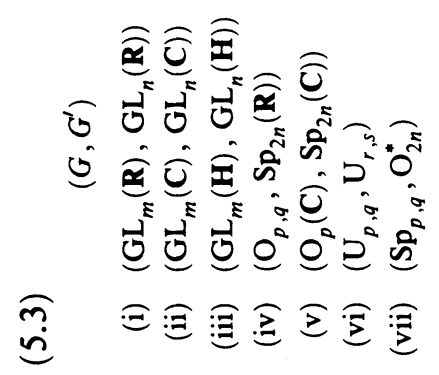


$M^{\prime(1,1)}$ will commute. (Since $K$ and $K^{\prime}$ commute, so do their linear spans, hence, so do the intersections of these spans with the unitary group.) Then verifying that $\left(M^{(1,1)}, M^{\prime(1,1)}\right)$ form a dual pair is a simple dimension check. Another way of expressing this is that if $W$ is given the complex structure corresponding to the unitary structure defining decomposition (2.1), then $K$ and $K^{\prime}$ generate mutual commutants inside $\operatorname{End}_{\mathbf{C}}(W)$. No doubt these facts can be established a priori.

In any case, Facts 1, 2, and 3 can be read off from (5.3), and there is perhaps some virtue in having the data it contains written down for reference.

\section{UNITARY VERSION}

The representation $\omega$ is, we noted at the beginning, a unitary representation of $\widetilde{\mathrm{Sp}}$. Theorem 1, however, takes no notice of this. It is couched in terms of the smooth vectors, and the elements of $\mathscr{R}(G, \omega)$ can and will be nonunitary. There is also a unitary version of Theorem 1 . It is not as precise, because as with most results involving $L^{2}$, it involves direct integrals and the consequent "almost everywhere" qualifications. This makes it inadequate for the purposes of automorphic forms. However, because of the intrinsic interest of the unitary case, and because of the cleanness of the result, we treat it.

Theorem 6.1. Let $\left(G, G^{\prime}\right) \subseteq \mathrm{Sp}$ be a reductive dual pair, and let $\left(\widetilde{G}, \widetilde{G}^{\prime}\right) \subseteq \widetilde{\mathrm{Sp}}$ be the inverse image pair in the metaplectic cover $\widetilde{\mathrm{Sp}}$ of $\mathrm{Sp}$. Then under the oscillator representation $\omega$ of $\widetilde{\mathrm{Sp}}$, the von Neumann algebras generated by $\omega(\widetilde{G})$ and $\omega\left(\widetilde{G}^{\prime}\right)$ are mutual commutants.

Proof. The proof is an adaptation of the proof of Theorem 2.1. Let $\overline{\mathscr{P}}$ denote the Hilbert space completion of the polynomials $\mathscr{P}$ endowed with the norm with respect to which $\mathfrak{s p}$ acts by skew-Hermitian operators. Similarly, let $\overline{\mathscr{Y}}^{(d)}$ and $\overline{\mathscr{Y}}^{\left(\tau, \tau^{\prime}\right)}$, etc. be the closure in $\mathscr{P}$ of the spaces $\mathscr{Y}^{(d)}, \mathscr{Y}^{\tau, \tau^{\prime}}$, etc. used in $\S 4$. In analogy with the definition given in (4.2), let us set

$$
\overline{\mathscr{X}}^{\tau, \tau^{\prime}}=\left(\mathscr{Y}^{\tau, \tau^{\prime}}+\mathscr{Y}^{(d)}\right)^{-} \mid \overline{\mathscr{Y}}^{(d)} \text {. }
$$

Then the image of $\mathscr{Y}^{\tau, \tau^{\prime}}$ in $\overline{\mathscr{Z}}^{\tau, \tau^{\prime}}$ is dense, and so the reasoning following (5.2) applies to show that

$$
\overline{\mathscr{Z}}_{\sigma, \sigma^{\prime}}=\left(L^{1}(\widetilde{G})^{\widetilde{K}} \cdot \mathscr{H}_{\sigma, \sigma^{\prime}}\right)^{-}=\left(L^{1}\left(\widetilde{G}^{\prime}\right)^{\widetilde{K}^{\prime}} \cdot \mathscr{H}_{\sigma, \sigma^{\prime}}\right)^{-} .
$$

Here $L^{1}(\widetilde{G})$ is the $L^{1}$ group algebra of $\widetilde{G}$ and $L^{1}(\widetilde{G})^{\widetilde{K}}$ is the subalgebra of elements that commute with $\widetilde{K}$. (Since we are now working with the full groups, we write $\widetilde{K}$, instead of $K$ as we did in $\S \S 3-5$.) Continuing, we conclude

$$
\bar{Z}_{\sigma, \sigma^{\prime}}=\left(L^{1}(\widetilde{G})^{\widetilde{K}}\left(e_{0}\right)\right)^{-}=\left(L^{1}\left(\widetilde{G}^{\prime}\right)^{\prime}\left(e_{0}\right)\right)^{-} .
$$

Now we need an analogue of Lemma 4.2. The most obvious analogue of Lemma 4.2 is false. However, there is an analogue which applies in our case. Let 
$A$ and $A^{\prime}$ be the von Neumann algebras generated by $L^{1}(\widetilde{G})^{\widetilde{K}}$ and $L^{1}\left(\widetilde{G}^{\prime} \widetilde{K}^{\prime}\right.$, respectively, by their actions on $\bar{Z}_{\sigma, \sigma^{\prime}}$. Then the well-known fact [D] that irreducible representations of $\widetilde{K}$ occur in irreducible representations of $\widetilde{G}$ with uniformly bounded multiplicity tells us that $A$ and $A^{\prime}$ are finite von Neumann algebras of type I. Thus, the following lemma applies.

Lemma 6.2. Let $A$ and $A^{\prime}$ be mutually commuting von Neumann algebras of finite type acting on a Hilbert space $\bar{Z}$. Suppose there is a vector $e_{0} \in \bar{Z}$ such that

$$
\bar{Z}=\left(A\left(e_{0}\right)\right)^{-}=\left(A^{\prime}\left(e_{0}\right)\right)^{-} .
$$

That is, $e_{0}$ is a cyclic vector for both $A$ and $A^{\prime}$. Then $A$ and $A^{\prime}$ are mutual commutants.

Proof. This follows directly from [Kd, Lemma 2].

I am grateful to $M$. Takesaki for this reference.

It follows, of course, from the lemma that $L^{1}(\widetilde{G})^{\widetilde{K}}$ and $L^{1}\left(\widetilde{G}^{\prime}\right)^{\widetilde{K}^{\prime}}$ generate mutual commutants on $\bar{Z}_{\sigma, \sigma^{\prime}}$. Since $\overline{\mathscr{Z}}_{\sigma, \sigma^{\prime}}$ is cyclic for $\overline{\mathscr{Z}}=\overline{\mathscr{X}}^{\tau, \tau^{\prime}}$, it follows [D] that $\widetilde{G}$ and $\widetilde{G}^{\prime}$ generate mutual commutants in $\bar{Z}$. We may assume by induction that $\widetilde{G}$ and $\widetilde{G}^{\prime}$ generate mutual commutants on $\overline{\mathscr{Y}}^{(d)}$. It is clear from Lemma 4.1 that $\overline{\mathscr{Z}}^{\tau, \tau^{\prime}}$ is characterized as a $\widetilde{G}$ submodule of $\left(\mathscr{Y}^{\tau, \tau^{\prime}}+\mathscr{Y}^{(d)}\right)^{-}$by the fact that all its subrepresentations contain the $\widetilde{K}$-type $\sigma$, but contain no $\widetilde{K}$-type $\tilde{\sigma}$ with $\operatorname{deg} \tilde{\sigma}<\operatorname{deg} \sigma$. Hence, the projection of $\left(\mathscr{Y}^{\tau, \tau^{\prime}}+\mathscr{Y}^{(d)}\right)^{-}$onto $\overline{\mathcal{X}}^{\tau, \tau^{\prime}}$ is in the von Neumann algebra generated by $\widetilde{G}$. (It will likewise be in the von Neumann algebra generated by $\widetilde{G}^{\prime}$.) From this we see that $\widetilde{G}$ and $\widetilde{G}^{\prime}$ generate mutual commutants on $\left(\mathscr{Y}^{\tau, \tau^{\prime}}+\mathscr{Y}^{(d)}\right)^{-}$. Repetition of this argument shows then that $\widetilde{G}$ and $\widetilde{G}^{\prime}$ generate mutual commutants on $\bar{y}^{(d+1)}$. Then Theorem 6.1 follows by a trivial induction.

\section{CONCLUDING REMARKS}

(a) In the proof of Theorem 2.1, a key role was played by the spaces $\mathscr{Z}^{\tau, \tau^{\prime}}$, defined by (5.2). The obvious next step in understanding the correspondence whose existence is established by Theorem 2.1 is a finer analysis of the spaces $\mathscr{Z}^{\tau, \tau^{\prime}}$. It seems conceivable that in some cases the action of $\mathscr{U}(\mathfrak{g})^{K}$ and of $\mathscr{U}\left(\mathfrak{g}^{\prime}\right)^{K^{\prime}}$ on the associated space $Z_{\sigma, \sigma^{\prime}}($ see $(4.8))$ is actually multiplicity free. It also seems possible that in some interesting cases $\mathscr{Z}^{\tau, \tau^{\prime}}$ may be irreducible as a $\mathfrak{g} \times \mathfrak{g}^{\prime}$ module. In any case, these spaces bear further study.

(b) Our analysis has shown that the bijection $\rho \leftrightarrow \rho^{\prime}$ between $\mathscr{R}(\widetilde{G}, \omega)$ and $\mathscr{R}\left(\widetilde{G}^{\prime}, \omega\right)$ has subordinated to it a bijection $\sigma \leftrightarrow \sigma^{\prime}$ between certain subsets of $\mathscr{R}(\widetilde{K}, \omega)$ and $\mathscr{R}\left(\widetilde{K}^{\prime}, \omega\right)$, defined by the action of $\widetilde{K} \cdot \widetilde{K}^{\prime}$ on the joint harmonics $\mathscr{H}(\widetilde{K}) \cap \mathscr{H}\left(\widetilde{K}^{\prime}\right)$. These corresponding representations $\sigma$ and $\sigma^{\prime}$ are the $\widetilde{K}$ (resp. $\widetilde{K}^{\prime}$ ) types of lowest degree (in the sense of degree in the 
polynomial ring $\mathscr{P}$ ) in the mutually corresponding representations of $\mathfrak{g}$ and $\mathfrak{g}^{\prime}$ occurring in $\mathscr{Z}^{\tau, \tau^{\prime}}$. These $K$-types of "lowest degree" will not in general be the "lowest $K$-type" in the sense of Vogan [V] or others, but there should be a strong relation between the two notions. It is not difficult to compute explicitly the correspondence $\sigma \leftrightarrow \sigma^{\prime}$ of simultaneous harmonics, but we will not do so here.

(c) The proof of Theorem 2.1 given here was suggested in part by the proof of the analogous result (see [H2] for a sketch) in the case of spherical representations for $p$-adic groups. In turn, this proof suggests a way of extending the $p$-adic results from the spherical case. This has been done in many cases. See [MVW, Wa]. This would then establish some global results about "lifting" in the theory of $\theta$-series.

\section{REFERENCES}

[C] P. Cartier, Quantum mechanical commutation relations and $\theta$-functions, Proc. Sympos. Pure Math., Vol. 9, Amer. Math. Soc., Providence, RI, 1966, pp. 361-383.

[D] J. Dixmier, Enveloping algebras, North-Holland, Amsterdam, New York, Oxford, 1977.

[F] D. Flath, Decomposition of representations into tensor products, Proc. Sympos. Pure Math., Vol. 32, Amer. Math. Soc., Providence, RI, 1979, pp. 179-184.

[G] S. Gelbart, Holomorphic discrete series for the real symplectic group, Invent. Math. 19 (1973), 49-58.

[GK] K. Gross and R. Kunze, Bessel functions and representation theory. II, J. Funct. Anal. 25 (1977), 1-49.

[Hl] S. Helgason, Differential geometry and symmetric spaces, Academic Press, New York, 1962.

[H1] R. Howe, Remarks on classical invariant theory, Trans. Amer. Math. Soc. (to appear).

[H2] _ Providence, RI, 1979, pp. 275-285.

[H3] _ _ Quantum mechanics and partial differential equations, J. Funct. Anal. 38 (1980), 188254.

[Kd] R. Kadison, Remarks on the type of von Neumann algebras of local observables in quantum field theory, J. Math. Phys. 4 (1963), 1511-1516.

[KV] M. Kashiwara and M. Vergne, On the Segal-Shale-Weil representation and harmonic polynomials, Invent. Math. 44 (1978), 1-47.

[MVW] C. Moeglin, M.-F. Vignéras, and J.-L. Waldspurger, Correspondences de Howe sur un corps p-adique, Lecture Notes in Math., no. 1291, Springer-Verlag, Berlin and New York, 1987.

[R] S. Rallis, On the Howe duality conjecture, Compositio Math. 51 (1984), 333-399.

[Sa] M. Saito, Représentations unitaires des groupes symplectiques, J. Math. Soc. Japan 24 (1972), 232-251.

[Sg] I. Segal, The complex-wave representation of the free boson field, preprint.

[Sh] D. Shale, Linear symmetries of free boson fields, Trans. Amer. Math. Soc. 103 (1962), 149-167.

[V] D. Vogan, The algebraic structure of the representations of semisimple Lie groups, Ann. of Math. (2) 109 (1979), 1-60.

[Wa] J. L. Waldspurger, preprint.

[WA] A. Weil, Sur certains groupes d'opérateurs unitaires, Acta Math. 111 (1964), 143-211.

[WH] H. Weyl, The classical groups, Princeton Univ. Press, Princeton, NJ, 1946.

Department of Mathematics, Yale University, New Haven, Connecticut 06520 